\title{
Dual plane multiple spatial watermarking with self-encryption
}

\author{
ROOPAM SADH* (D), NISHCHOL MISHRA and SANJEEV SHARMA \\ School of Information Technology, Rajiv Gandhi Proudyogiki Vishwavidyalaya, Bhopal 462036, India \\ e-mail: roopam.sadh@gmail.com; nishchol@rgtu.net; sanjeev@ rgtu.net
}

MS received 11 February 2014; revised 10 May 2015; accepted 30 August 2015

\begin{abstract}
Watermarking has established itself as a promising solution in the context of digital image copyright protection. Frequency domain watermarking is mainly preferred due to associated robustness and perceptual issues but requires a large amount of computation. On the other hand spatial domain watermarking is much faster but lacks when robustness is concerned. In this paper a block based spatial domain watermarking technique for color images is proposed that is fast and robust. Two-way embedding introduced in the paper provides capability to previously estimate perceptual degradation in host image due to watermark embedding. Multiple watermarking in two component images is used to achieve better imperceptibility and better robustness. Self-encryption introduced aims to remove dependency over external secret key however optional encryption with random secret keys is proposed for providing better security. Proposed embedding technique is an effort to achieve proper balance between robustness and quality degradation of image. Experiments suggest proposed method is robust against processing attacks such as spatial filtering transformations image enhancement and loss compression.
\end{abstract}

Keywords. Spatial domain watermarking; multiple watermarking; dual plane watermarking; copyright protection; selfencryption.

\section{Introduction}

Today, most of the internet traffic includes multimedia contents. In many of the industries like art work industries, digital media is used as product or service. In such domains illegal access, illegal distribution and altercation of digital media are serious challenges. That is why protection of ownership rights has become the key issue. Digital watermarking is one of the most widely used ownership right protection techniques [1].

In watermarking an unobtrusive mark or label that is sufficient to represent the identity of owner is embedded into image and at the time of copyright claim owner shows his ownership by extracting watermark [2]. For a good watermarking technique some of the key concerns are as such: imperceptibility, secrecy of watermark, robustness of marking method, efficient embedding and extraction procedures [3]. Imperceptibility and robustness against attacks are contradictory issues so great balance of both is desired in watermarking

Watermarking can be classified in two broad categories based on their working domains; spatialdomain and frequency domain [4]. Watermarking in frequency domain shows more robustness but complexity and computational cost of forward and inverse transforms can be termed as their drawbacks Moreover in frequency domain methods the size

*For correspondence of watermark is restricted to preserve the quality of watermarked image. Spatial domain watermarking methods on the other hand are comparatively very fast but they hinder when robustness is concerned [5]. In this paper a block based spatial domain watermarking is proposed in which two component planes of color host image are used for embedding purpose. Embedding of watermark is done by two-way mechanism introduced in this paper. Two levels of encryption are used for watermark security. At first level an automatic self-encryption procedure is introduced that produces keys with the help of host image information and encrypt the watermark. Optional random key encryption is used for addition security in second level of encryption. Proposed method is robust and gives better performance than other spatial domain watermarking methods.

Rest of the paper is organized as follows: section 2 describes literature survey of previously designed watermarking methods. Section 3 describes proposed watermarking scheme with encryption, embedding and extraction given in subsections. Section 4 explains experimental results and conclusion is given in section 5

\section{Literature survey}

Frequency domain watermarking is mainly used for the purpose of robust watermarking [6]. Sánchez et al [7] proposed a frequency domain watermarking in which DCT is applied on $8 * 8$ image blocks; watermark is embedded bit by 
bit through marking the DCT block coefficients under the threshold. Generally low frequency coefficients are modified for better resistance against attacks. Raval et al [8] uses DWT to embed multiple watermarks for resisting both loss compression and filtering attacks. The transform is divided into low frequency band and high frequency band then two different watermarks are embedded in these two bands. This method performs well against filtering attacks. Cheng et al [9] inserted watermark into host image by randomly selected location in DCT and this makes the watermarking more resistant against low-pass, highpass and median filtering. Bhatnagar \& Raman [10] suggests a watermarking method in which Hilbert space filling curve is used to find significant blocks of host image in order to construct reference image. Fractional Fourier Transform (FrFT) followed by singular value decomposition (SVD) is applied on reference image. Singular values of watermark are used to modify singular values of reference image. Inverse SVD followed by inverse FrFT is performed to find modified reference image. Now blocks are placed to their original positions to obtain watermarked image. Watermarking scheme proposed by Chavan et al [11] applies combination of DWT and block wise DCT on color host image and block wise DCT on watermark. Embedding is done by replacing low frequency DCT coefficients of watermark blocks with mid-frequency DCT coefficients of host image blocks. Method is fairly robust against JPEG compress, salt and pepper noise but is not robust against geometric distortions like rotation, translation and scaling.

Spatial domain watermarking techniques are mainly used for fragile watermarking [6]. One of the most conventional methods of spatial watermarking is LSB substitution technique, [12] in which least significant bits of cover image are replaced by the bits of watermark. This method is not secure because attacker can replace least significant bits of watermarked image by randomly chosen bits. Nikolaidis \& Pitas [13] proposed a blind watermarking method in which watermark is embedded by varying the intensities of randomly chosen pixels of host image. For increasing the robustness, Nikolaidis \& Pitas [13] generalized this scheme by using blocks instead of pixels. Main problem with this method is that binary watermark can consist of equal number of zeros and ones, no other choice is possible and method is not fairly robust. In watermarking scheme proposed by Maity \& Kundu [14] position of homogenous blocks (based on small value of variance) in original image is noted in a two dimensional binary matrix called secret image. Watermark bit is inserted by replacing integer part bit of average value of homogenous blocks and insertion position is recorded in secret image. This secret image is used for extraction purpose. This method is not robust against random noise addition, cropping, rotation and JPEG compression. Kimpan et al [15] proposed idea of variable size blocks. Embedding is done by modifying brightness level of each block according to watermark bit either or 1. Larger blocks are subdivided into sub-blocks and brightness of pixels of each sub-block is modified according to the mean intensity of pixels in sub-blocks. Lopes et al [16] suggested embeds watermark in edge pixels of host image based on texture analysis done in spatial domain. Image is divided into the blocks of $8 * 8$ and these blocks are subdivided according to texture analysis (either edge pixel or not). Now embedding of watermark can be done in either frequency or spatial domain. Verma et al [17] proposed a block based method for color images in which blue component of color image is divided into blocks of size $8 * 8$ and intensity of each pixel in the block is modified either by increasing the intensity (if the bit of watermark is 1 ) or by decreasing (if the bit is 0 ) with predefined value. Before embedding watermark is encoded by convolution encoding. Method is not fairly robust against cropping attack because single watermark is embedded in whole image. Cika [18] proposed a similar block based method in which Pseudo random key of equal size is XORed with binary watermark to produce encrypted watermark. Watermark is embedded in both red and blue components of image. Embedding is done by same procedure as reported in Verma et al [17]. This method also lacks in robustness when attacked with cropping. For removing this deficiency. Nasir et al [19] proposed concept of multiple spatial watermarking. In which watermark is divided into four parts and each part is encrypted separately. These parts are embedded in different regions of blue component image of color host image. Embedding is done by same procedure as in Verma et al [17]. This method shows excellent resiliency against a number of processing attacks such as cropping, filtering and geometrical transformation.

Though spatial domain watermarking method proposed by Nasir et al [19] is fairly robust but it leaves some questions unanswered that are as follows

- How to modify pixel intensities that lie on fixed range boundary (0 to 255)?

- How to predict and control quality degradation of image due to watermark embedding?

- How to handle loss compression with high compression ratio?

Taking these issues under consideration; a block based spatial domain watermarking technique is proposed. It can be said as the extension of method proposed by Nasir et al [19].

\section{Proposed watermarking scheme}

The overall description of proposed watermarking procedure can be summarized as follows: color host image is divided into its component planes in which green and blue components are used for embedding purpose. Method first encrypts the watermark by self-encryption. Optional random secrete key based encryption can be used for additional security purpose, if required. Embedding procedure divides the host component planes into regions and divides encrypted 
watermark into four parts. These watermark parts are then embedded into different regions of component planes. Both watermarked planes together with the original red plane are merged in order to obtain watermarked color image. At the time of extraction, comparison between pixel intensities of original image and watermarked image takes place. All copies of watermark parts are extracted, in which the best four copies that create whole watermark are chosen. Whole method is illustrated by figure 1 . Whole method can be broadly divided into four parts that are encryption, embedding, extraction and decryption.

\subsection{Encryption procedure}

In proposed scheme encryption procedure can be divided into two parts as follows:

\section{(1) Self-encryption}

(2) Random secrete key encryption (optional).

Use of two-level encryption is mainly intended to answer the two different kinds of user needs. First is fast and automatic procedure with no need of providing external keys and second is providing security as much as possible with the keys provided from user. So the use of two-level encryption is dependent on user preference for ex-general social networking user may require watermarking but with less computational costs and with no compulsion of manually providing and maintaining different secrete keys. This type of user can be benefitted by using self-encryption and skipping random key encryption. On the other hand for a professional photo artist high security of watermark is primary issue. So this kind of user can use both levels of encryption to match his need. The purpose of self-encryption is to make the watermark unreadable and freeing up the owner from the burden of maintaining secrete keys for uncertain period of time. Self-encryption is an automatic procedure as it does not require outside keys instead it creates secret keys with the help of host image information. Optional random secret key encryption module is provided for occasions when more image security is demanded from user. It requires four different pseudo random binary keys to be provided by user at the time of encryption and decryption. User has to set his preference to use random key encryption before applying watermarking.

3.1a Selfencryption: Self-encryption works in two phases; first phase is generation of secrete keys and second phase is encryption of watermark. Choice of mathematical function to produce secrete-keys and the size of watermark are both dependent on the size of image being watermarked. We have assumed host image of size $512 * 512$, watermark size is of $32 * 32$ and average intensity value as secrete key. In embedding procedure section the basis of these assumptions is made clear. In phase-1, blue plane (choice

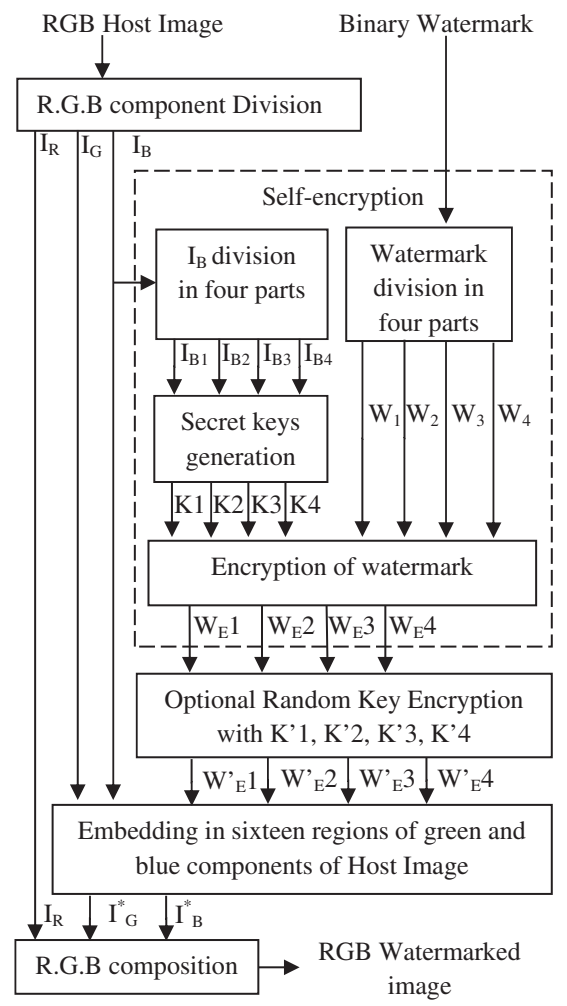

(a)

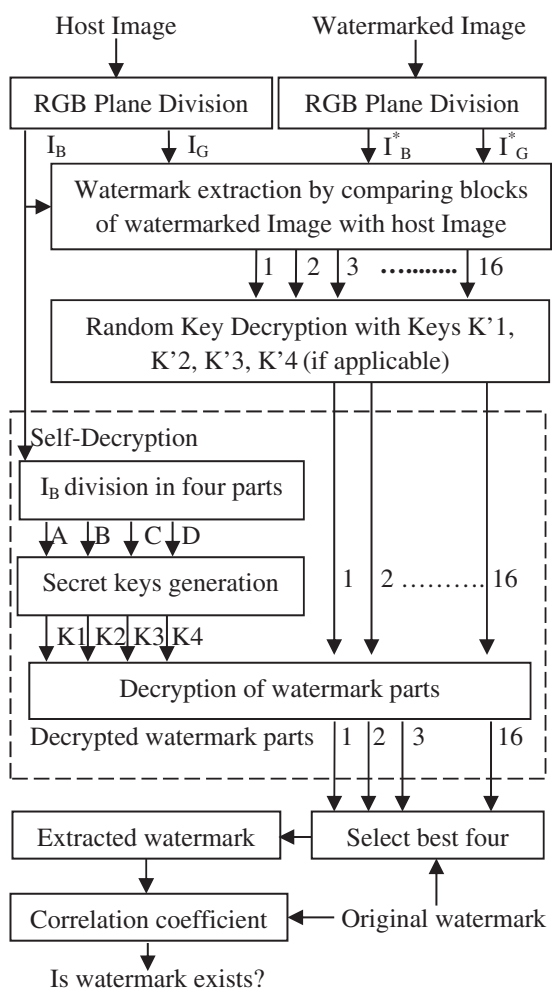

(b)

Figure 1. (a) Watermark embedding procedure; (b) Watermark extraction. 
is random) of host image is taken and divided into four equal non-overlapping parts. Average intensity value of each part is treated as the secrete key. By this way four different secrete keys are calculated and these secret keys are represented by $\mathrm{K} 1, \mathrm{~K} 2, \mathrm{~K} 3, \mathrm{~K} 4$ respectively. Average intensity values (secret keys) lie in the range of 0-255 and value under this range is required by self-encryption procedure (phase-2) as this value will represent a specific binary cell location between 256 possible binary cell locations of a binary watermark part of size $16 * 16$.

Next task is to encrypt binary watermark with these generated keys (phase-2). Binary watermark is taken because proposed watermarking method is block based spatial domain method and this type of methods works by modifying pixel intensities of the block. Binary value 0 or 1 can be represented by decreasing the intensity or by increasing the intensity respectively. Binary watermark image of size $32 * 32$ is divided into four parts of size $16^{*} 16$ and they are represented by W1, W2, W3, and W4. Each 16*16 sized watermark part represents the matrix of degree $16^{*} 16$ (containing a total of 256 locations). We assume that the cells of matrix are numbered sequentially, like cell $(1,1)$ is numbered as 1 , cell $(1,2)$ as $2,(2,1)$ as 17 and so on. Encryption method simply arranges the binary values inside the matrix cells in diagonal fashion. After arranging the values in that way, cell location which is equal to the secret key is treated as the first cell of encrypted watermark and again the matrix is rearranged. Figure 2 demonstrates this procedure of self-encryption (phase-2) by taking example matrix of $4 * 4$ (watermark part) and example secret key value 11 .
Outcomes of the encryption that have taken place four times, each time for one of the watermark parts are represented by $\mathrm{W}_{\mathrm{E}} 1, \mathrm{~W}_{\mathrm{E}} 2, \mathrm{~W}_{\mathrm{E}} 3$, and $\mathrm{W}_{\mathrm{E}} 4$. Whole procedure of Self-encryption is illustrated by figure 3 .

3.1b Optional random key encryption: Self-encryption is just a way to make marking information unreadable and it is less secure because key space of self-encryption is small (equal to the number of binary cell location in watermark parts). Random key encryption can be used for providing additional security (increasing the key space) of watermark and to make watermark extraction process owner dependent. Four pseudo random binary bit sequences of size $16^{*} 16$ denoted by $\mathrm{K}^{\prime} 1, \mathrm{~K}^{\prime} 2, \mathrm{~K}^{\prime} 3$ and $\mathrm{K}^{\prime} 4$ are used as secrete keys. These secrete keys are then XORed (exclusive OR) on bit level with four self-encrypted watermark parts that are produced as the output of self-encryption procedure i.e. each bit of $\mathrm{K}^{\prime} 1$ is XORed with corresponding bit of $\mathrm{W}_{\mathrm{E}} 1$. This can be represented by the following:

$K^{\prime} 1(i, j) \oplus W_{E} 1(i, j) \quad$ (where $(i, j)$ is the binary cell location such that $1<=i, j<=16$ ).

In the same way $\mathrm{K}^{\prime} 2$ is XORed with $\mathrm{W}_{\mathrm{E}} 2, \mathrm{~K}^{\prime} 3$ is XORed with $\mathrm{W}_{\mathrm{E}} 3$ and $\mathrm{K}^{\prime} 4$ is XORed with $\mathrm{W}_{\mathrm{E}} 4$. Outcomes of this process are encrypted watermark parts $\mathrm{W}_{\mathrm{E}}^{\prime} 1, \mathrm{~W}_{\mathrm{E}}^{\prime} 2, \mathrm{~W}_{\mathrm{E}}^{\prime} 3$ and $\mathrm{W}_{\mathrm{E}}^{\prime} 4$. A similar approach of encryption is also used in Cika [18]. Random key encryption is sufficiently secure as it uses four random binary sequences of size $16^{*} 16$ (the key

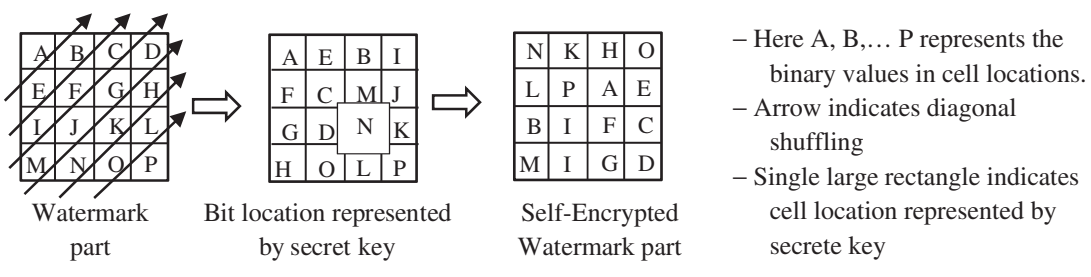

Figure 2. Demonstration of self-encryption phase.
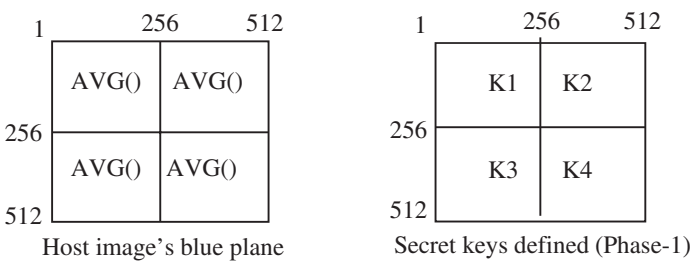

$$
\begin{aligned}
\operatorname{AVG}()= & \text { Average intensity } \\
\mathrm{SE} & =\text { Secret key } \\
\mathrm{W} & =\text { Binary watermark } \\
\mathrm{W}_{\mathrm{E}} & =\text { Encrypted watermark } \\
& 1<=\mathrm{i}<=4
\end{aligned}
$$

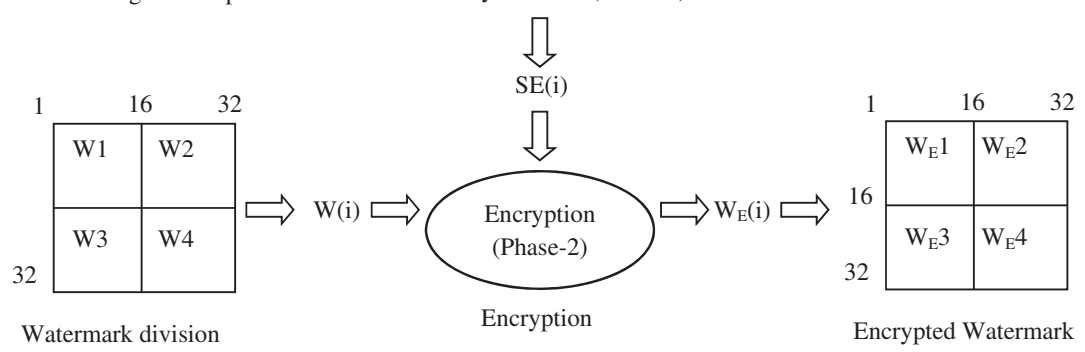

Figure 3. Overall self-encryption procedure. 
space is large enough, $2^{256}$ for a single random binary bit sequence). Combination of self-encryption and random key encryption makes the proposed encryption highly secure.

\subsection{Embedding procedure}

Embedding is done in green and blue planes of host image for embedding purpose. Blue plane is chosen because human eyes are less sensitive to changes made in blue plane and green plane is chosen as it shows more endurance against JPEG compression [20]. These two planes are divided into equal sized regions. One single region will be embedded with one part of encrypted watermark information. This is done by dividing region into $8 * 8$ sized blocks and embedding one bit of information in each block. Reason for choosing block size as $8 * 8$ is that $8 * 8$ is also the size of JPEG blocks so the JPEG compression will affect each watermarked bit independently [5]. Size of the region is taken $128 * 128$ as 128 is the exact divisor of 512 which is assumed to be the size of our test image in both the direction and also supports the concept of multiple watermarking [19] efficiently i.e. by taking region of size $256 * 256$ multiple watermarking cannot be achieved because at least four regions are needed for holding single complete copy of encrypted watermark. Size of the region also forms the basis of choosing the size of watermark; as in our case region size $128 * 128(16 * 8$ by $16 * 8)$ defines block size of $8 * 8$ and a part of encrypted watermark of the size $16 * 16$. This is the reason behind choosing binary watermark of size $32 * 32$.

Four encrypted watermark parts $\mathrm{W}_{\mathrm{E}}^{\prime} 1, \mathrm{~W}_{\mathrm{E}}^{\prime} 2, \mathrm{~W}_{\mathrm{E}}^{\prime} 3$ and $\mathrm{W}_{\mathrm{E}}^{\prime} 4$ are now represented by $(1,2,3,4)$ for the sake of convenience. Host image $\mathrm{I}$ is divided into its component Images $\mathrm{I}_{\mathrm{R}}, \mathrm{I}_{\mathrm{G}}, \mathrm{I}_{\mathrm{B}}$ such that $I_{R}(i, j), I_{G}(i, j)$ and $I_{B}(i, j)$ are in the range [0 255] (where $(i, j)$ is the pixel location such that $0<i, j<=512$ ).

Components $\mathrm{I}_{\mathrm{G}}$ and $\mathrm{I}_{\mathrm{B}}$ are divided into regions. Regions are selected for embedding of watermark parts $(1,2,3$, and 4) according to figure 4.

Block is used to embed the single bit (either 0 or 1 ) of encrypted watermark part. 0 bit is embedded in $I_{B}$ and 1 bit is embedded in $\mathrm{I}_{\mathrm{G}}$. Pixel intensities in each block are slightly altered with embedding strength $\alpha$ by the help of two-way mechanism. Value of $\alpha$ is user dependent. High value of $\alpha$ improves the robustness of watermarking but it causes increased level of noise in host image due to watermark
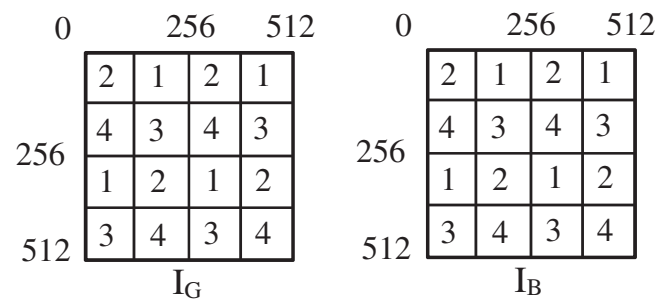

Figure 4. Division of Host image components into regions. embedding and degradation of image quality can be perceptually visible to the viewer, especially when the image is broken into its component planes. So the value should be chosen intelligently. We have taken $\alpha=5$ as suggested by Verma et al [17] and Nasir et al [19]. When embedding bit 0 in $\mathrm{I}_{\mathrm{B}}$; if pixel intensity under present consideration is greater than $\alpha$; then it is decreased by $\alpha$ or if intensity is less than $\alpha$ then it is increased with $\alpha$. Same procedure is done for bit 1 which is embedded in $\mathrm{I}_{\mathrm{G}}$; if the intensity of current pixel is less than 255- $\alpha$ then it is increased with $\alpha$ else it is decreased with $\alpha$. This two-way embedding mechanism prevents pixel values to go outside their range 0-255. That is why behavior of every pixel after watermarking is predictable and this makes proposed method capable to claim a certain amount of introduced noise due to watermark embedding. Another benefit of using two components is better imperceptibility as compared to Nasir et al [19] (using single blue plane) because difference between marked and unmarked pixels remains $\alpha$ in each plane. Proposed embedding can be described as follows.

$$
\begin{aligned}
\text { IF } W_{E}(i, & j)=1 \quad(1<=i, j<=8) \\
& \text { IF } I_{G}(i, j)<=(255-\alpha) \\
& I_{G}^{*}(i, j)=I_{G}(i, j)+\alpha \\
& \text { ELSE } \\
& I_{G}^{*}(i, j)=I_{G}(i, j)-\alpha
\end{aligned}
$$

ELSE

$$
\begin{aligned}
& \text { IF } I_{B}(i, j)>=\alpha \\
& I_{B}^{*}(i, j)=I_{B}(i, j)-\alpha ; \\
& \text { ELSE } \\
& I_{B}^{*}(i, j)=I_{B}(i, j)+\alpha ;
\end{aligned}
$$

where $(i, j)$ are pixel locations, $\mathrm{W}_{\mathrm{E}}$ represents pixel of encrypted watermark part (i.e. $\mathrm{W}_{\mathrm{E}}^{\prime} 1$, etc.). $I_{G}^{*}(i, j), I_{B}^{*}(i, j)$ represents watermarked intensity pixel of green and blue plane respectively. $I_{G}(i, j), I_{B}(i, j)$ represents original host pixel of green and blue plane respectively. Finally $I_{G}^{*}, I_{B}^{*}$ together with red plane of original host image $\left(\mathrm{I}_{\mathrm{R}}\right)$ are combined to make the watermarked image $\mathrm{I}^{*}$.

$$
I^{*}=I_{R}+I_{G}^{*}+I_{B}^{*}
$$

\subsection{Extraction process}

Proposed method is nonblind hence it requires original host image at the time of extraction process. Extraction is done through finding the difference between pixel intensity values of original image blocks and watermarked image blocks in both planes (extraction of watermark takes place by analyzing the results of difference in pixel intensity values in both 
blue and green planes separately in parallel fashion). The difference between pixel intensities of original block and watermarked block either positive or negative shows possibility of a single watermark bit i.e. a watermarked bit could be if difference is found in blue plane and watermark bit could be 1 if difference is found in green plane. If no difference found then it could be 1 in blue plane and could be in green plane. By this way all the pixels of the same block are analyzed and a decision is made about the possibility of bit to be 0 or 1 that is represented by the block on the basis of indication given by majority of pixels. For taking this decision probabilistic calculation is used.

Original host image $\mathrm{I}$ and watermarked image $\mathrm{I}^{*}$ are divided into their component images $\left(\mathrm{I}_{R}, \mathrm{I}_{\mathrm{G}}, \mathrm{I}_{\mathrm{B}}\right.$ and $\mathrm{I}_{\mathrm{R}}^{*}, \mathrm{I}_{\mathrm{G}}^{*}, \mathrm{I}_{\mathrm{B}}^{*}$ respectively). Green and blue planes of both input images are divided into regions of $128 * 128$ and each region is further divided into blocks of size $8 * 8$. At first probabilities of a watermark bit to be either 0 or 1 in blue plane denoted by $\mathrm{P}_{\mathrm{BZERO}}, \mathrm{P}_{\mathrm{BONE}}$ and for green plane $\mathrm{P}_{\mathrm{GZERO}}, \mathrm{P}_{\mathrm{GONE}}$ respectively are calculated. All four probability parameters are first initialized to zero. Now each pixel intensity of block from $\mathrm{I}_{\mathrm{G}}$ is compared with $\mathrm{I}_{\mathrm{G}}^{*}$ and each pixel intensity of block from $\mathrm{I}_{\mathrm{B}}$ is compared with $\mathrm{I}_{\mathrm{B}}^{*}$ to find values of these probabilities. During the time of watermark embedding we modified the pixel intensity values of host image block with embedding strength $\alpha$. Now at the time of extraction we observe that either the difference between pixel intensity of host image and watermarked image is greater than $\alpha-1$ or not. We use $\alpha-1$ instead of $\alpha$ because we assume that a small amount of change in intensity of pixels can be possible due to accidental or intentional modification of image information e.g. attacks such as smoothening, loss compression and transmission errors. The process of finding values of these probability parameters is described by the following algorithm.

For green plane -

$$
\begin{gathered}
\text { IF }\left(I_{G}(i, j) \leq 255-\alpha\right) \\
\left(\text { Calculation of } \mathrm{P}_{\mathrm{GZERO}} \text { and } \mathrm{P}_{\mathrm{GONE}}\right) \\
\text { IF }\left(I_{G}^{*}(i, j)-I_{G}(i, j)\right) \geq \alpha-1 \\
\mathrm{P}_{\mathrm{GONE}}=\mathrm{P}_{\mathrm{GONE}}+1 / 64 ;
\end{gathered}
$$

\section{ELSE}

$$
\mathrm{P}_{\mathrm{GZERO}}=\mathrm{P}_{\mathrm{GZERO}}+1 / 64 ;
$$

\section{ELSE}

$$
\begin{aligned}
& \text { IF }\left(I_{G}(i, j)-I_{G}^{*}(i, j)\right) \geq \alpha-1 \\
& \mathrm{P}_{\mathrm{GONE}}=\mathrm{P}_{\mathrm{GONE}}+1 / 64
\end{aligned}
$$

ELSE

$$
\mathrm{P}_{\mathrm{GZERO}}=\mathrm{P}_{\mathrm{GZERO}}+1 / 64 ;
$$

For blue plane -

$$
\begin{aligned}
& \text { IF }\left(I_{B}(i, j) \geq \alpha\right) \quad 0<i, j \leq 8 \\
& \text { (Calculation of } \mathrm{P}_{\mathrm{BZERO}} \text { and } \mathrm{P}_{\mathrm{BONE}} \text { ) } \\
& \text { IF }\left(I_{B}(i, j)-I_{B}^{*}(i, j)\right) \geq \alpha-1 \\
& \mathrm{P}_{\mathrm{BZERO}}=\mathrm{P}_{\mathrm{BZERO}}+1 / 64 ; \\
& \text { ELSE } \\
& \mathrm{P}_{\mathrm{BONE}}=\mathrm{P}_{\mathrm{BONE}}+1 / 64 ; \\
& \text { ELSE } \\
& \text { IF }\left(I_{B}^{*}(i, j)-I_{B}(i, j)\right) \geq \alpha-1 \\
& \mathrm{P}_{\mathrm{BZERO}}=\mathrm{P}_{\mathrm{BZERO}}+1 / 64 ; \\
& \mathrm{P}_{\mathrm{BONE}}=\mathrm{P}_{\mathrm{BONE}}+1 / 64 ;
\end{aligned}
$$

As described by the algorithm each time a pixel position in a block (64 pixel locations) shows the difference near the value of $\alpha$ in green plane; the probability of a bit being a 1 is increased by a factor of $1 / 64$ otherwise probability of bit being a 0 is increased by same amount. Identical procedure is followed for blue plane. For a single bit position of watermark four values of probability are calculated; two from blue plane and two from green plane i.e. $\mathrm{P}_{\mathrm{GONE}}, \mathrm{P}_{\mathrm{BONE}}$ for the

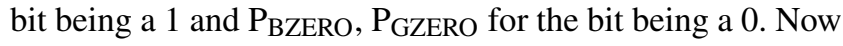
higher value in each pair is selected; for example - higher value between $\mathrm{P}_{\mathrm{GONE}}, \mathrm{P}_{\mathrm{BONE}}$ is preferred for a bit being a 1. Higher value in each pair is selected and these two outcomes are denoted by two different variables namely $P_{\text {ZERO }}$ and $\mathrm{P}_{\mathrm{ONE}}$. This is described as follows:

$$
\begin{array}{r}
\mathbf{I F}\left(\mathrm{P}_{\mathrm{GONE}} \geq \mathrm{P}_{\mathrm{BONE}}\right) \\
\mathrm{P}_{\mathrm{ONE}}=\mathrm{P}_{\mathrm{GONE}} ;
\end{array}
$$

\section{ELSE}

$$
\begin{gathered}
\mathrm{P}_{\mathrm{ONE}}=\mathrm{P}_{\mathrm{BONE}} ; \\
\mathbf{I F}\left(\mathrm{P}_{\mathrm{GZERO}} \geq \mathrm{P}_{\mathrm{BZERO}}\right) \\
\mathrm{P}_{\mathrm{ZERO}}=\mathrm{P}_{\mathrm{GZERO}} ;
\end{gathered}
$$

ELSE

$$
\mathrm{P}_{\mathrm{ZERO}}=\mathrm{P}_{\mathrm{BZERO}}
$$

Now extracted watermark bit either 0 or 1 for current location is decided by comparing variables $\mathrm{P}_{\mathrm{ONE}}$ and $\mathrm{P}_{\mathrm{ZERO}}$.

$$
\begin{aligned}
\mathbf{I F}\left(\mathrm{P}_{\mathrm{ZERO}}\right. & \left.>\mathrm{P}_{\mathrm{ONE}}\right) \\
& W_{E}^{*}(i, j)=0 ;
\end{aligned}
$$

\section{ELSE}

$$
W_{E}^{*}(i, j)=1
$$


Here $\mathrm{W}_{\mathrm{E}}^{*}$ is the extracted watermark.

By following this procedure iteratively for all the blocks in a region an encrypted watermark part is created (since a region is embedded with an encrypted watermark part at the time of embedding). In the same way as described above, all the regions of watermarked image are processed to find every encrypted watermark parts that were embedded during embedding. Now each copy of encrypted watermark parts extracted from different regions of watermarked image is given to decryption function.

\subsection{Decryption procedure}

If random key encryption was used then user is required to provide four pseudo random binary keys $K^{\prime} 1, K^{\prime} 2, K^{\prime} 3$ and

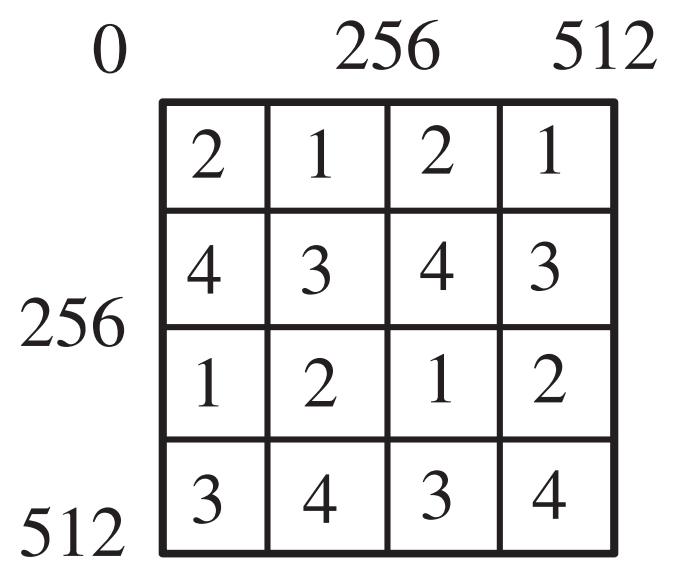

Figure 5. Positions of watermark parts in component images.
$\mathrm{K}^{\prime} 4$ that were used while encrypting the watermark. Since watermark was divided into four parts and each single part is embedded in many different regions hence all similar copies of an extracted watermark part would be decrypted with same random sequence. Positions of embedded watermark parts in watermarked image are given in figure 5.

Sequences $\left(K^{\prime} 1, K^{\prime} 2, K^{\prime} 3\right.$ and $\left.K^{\prime} 4\right)$ are XORed with respective extracted watermark parts for example- watermark part 2 is XORed with $\mathrm{K}^{\prime} 2$. After completing this procedure for all copies of each watermark part; outcomes of this decryption process are then given to Self-decryption function with host image.

Now original host image is divided into its component planes. Blue plane is divided into four parts in order to find four secret keys (K1, K2, K3 and K4) for self-encryption. Process of finding secret keys is same as described in self-encryption procedure. Similar copies of each part are decrypted by their corresponding keys.

After decryption best of four similar copies of the same part is chosen on the basis of matching with corresponding part of original watermark. For doing this original watermark is divided into four parts (W1, W2, W3, W4). The matching is done by using a counter that is incremented each time when a match is found for each bit location. The copy having highest value of this counter is selected to produce extracted watermark. By this way all best copies are selected and combined to produce whole extracted watermark. Figure 6 illustrates self-decryption of a watermark part 1 and matching process.

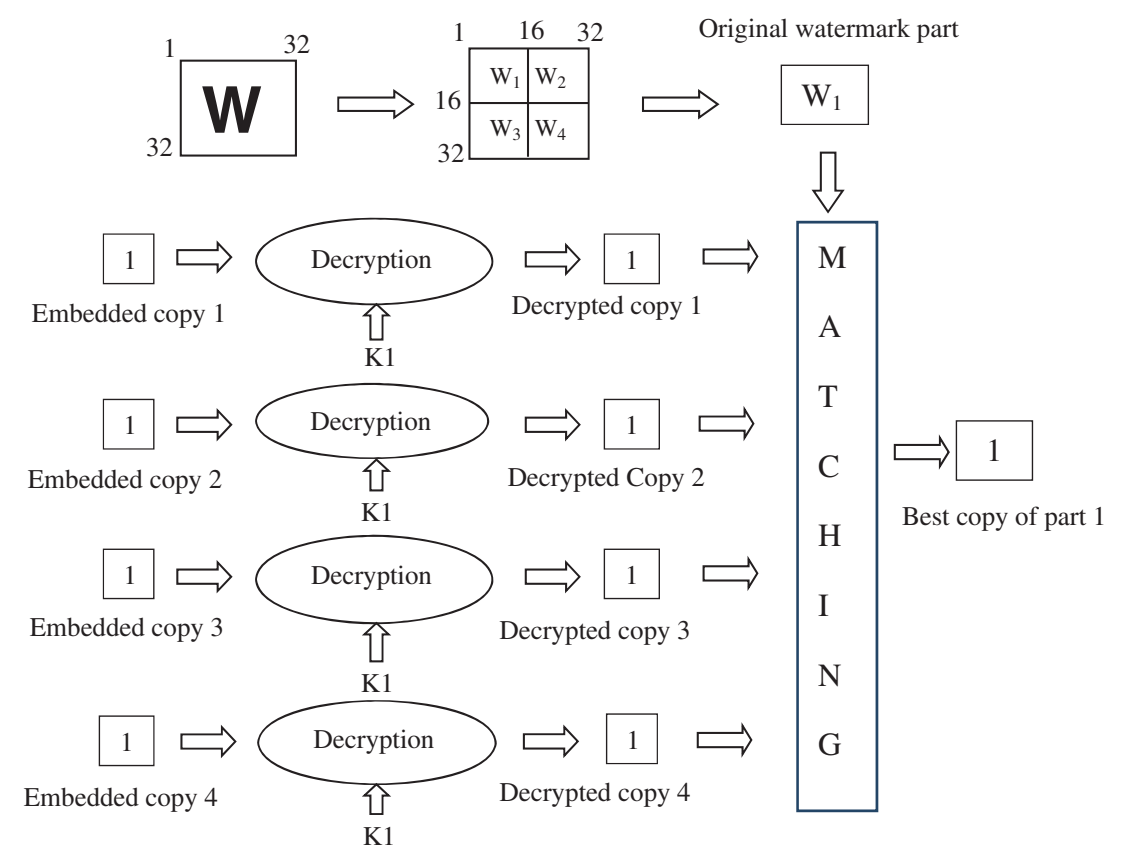

Figure 6. Demonstration of self-decryption and matching process taking watermark part 1 . 


\section{Experimental results}

Performance of watermarking is tested by two parameters. One is additive noise due to watermark embedding and second is similarity of extracted watermark with original watermark. PSNR is used for calculating additive noise due to proposed watermark embedding.

$$
\begin{aligned}
P S N R= & 10 \log _{10} \frac{(255)^{2}}{M S E} \\
M S E= & \frac{1}{(3 \times m \times n)} \sum_{i=1}^{m} \sum_{j=1}^{n}\left[\left(x_{r}(i, j)-y_{r}(i, j)\right)^{2}+\right. \\
& \left.\left(x_{g}(i, j)-y_{g}(i, j)\right)^{2}+\left(x_{b}(i, j)-y_{b}(i, j)\right)^{2}\right]
\end{aligned}
$$

where red, green, and blue component pixels of original image are represented by $x_{r}(i, j), x_{g}(i, j), x_{b}(i, j)$ respectively. And $y_{r}(i, j), y_{g}(i, j), y_{b}(i, j)$ represents red, green and blue component of watermarked pixel at location $(i, j)$. $m \times n$ is the size of both images.

Similarity between original and extracted watermark is calculated by CC (correlation-coefficient).

$$
C C=\frac{\sum_{i} \sum_{j}\left(W(i, j)-\overline{m_{w}}\right)\left(W *(i, j)-\overline{m_{W}^{*}}\right)}{\sqrt{\sum_{i} \sum_{j}\left(W(i, j)-\overline{m_{w}}\right)^{2}} \sqrt{\sum_{i} \sum_{j}\left(W *(i, j)-\overline{m_{w}^{*}}\right)^{2}}}
$$

where $W(i, j), W *(i, j)$ is original and extracted watermark bit respectively at $(i, j)$ location. $\overline{m_{w}}$ and $\overline{m_{w}^{*}}$ are mean values of original and extracted watermark respectively. CC can have a maximum value of 1 .

Test images of size $512 \times 512$ are taken from CVGUGR test image database CVG-UGR test image database at [21]. Binary watermark is of the size $32 \times 32$. Embedding strength $\alpha$ is taken as 5, as suggested by Verma et al [17] and Nasir et al [19]. Moreover it is found as suitably balanced value to achieve imperceptibility and robustness in experiments. Experimental results for imperceptibility with different values of $\alpha$ are shown in figure 7 under the section Imperceptibility test. Attacks defined in Stirmark bench 4.0 [22] are performed on test images for checking the performance. Stirmark benchmark 4.0 attacks [23] are depicted in table 1 .

Table 1. Stirmark benchmark 4.0.

\begin{tabular}{lc}
\hline Attacks & Level \\
\hline Affine transform & $1,2,3,4,5,6,7,8$. \\
Gaussian filtering & 1,2 \\
Cropping (\%) & $25,50,75$ \\
JPEG (\%) & $20,30,40,50,60,70,80$ \\
Median filtering & $3,5,7,9$ \\
Noise (\%) & $10,20,30,40,50,60,70,80$ \\
Scale (\%) & $50,75,90,125,150,200$ \\
Remove lines & 10 to $100($ step of 10$)$ \\
Rotation crop & $-2,-1,-0.5,0.5,1,2$ \\
Rotation scale & $-2,-1,-0.5,0.5,1,2$ \\
Rotation & $-2,-1,0.5,1,2,5,10,25,90$ \\
\hline
\end{tabular}

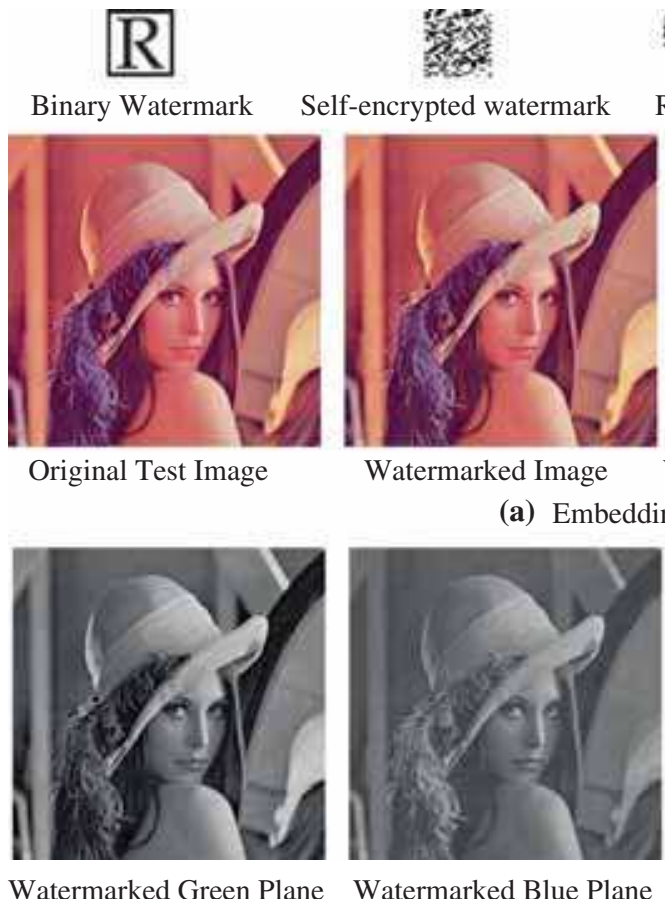

(b) Embedding strength $\alpha=3$

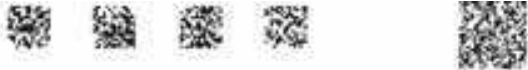

Random binary sequences
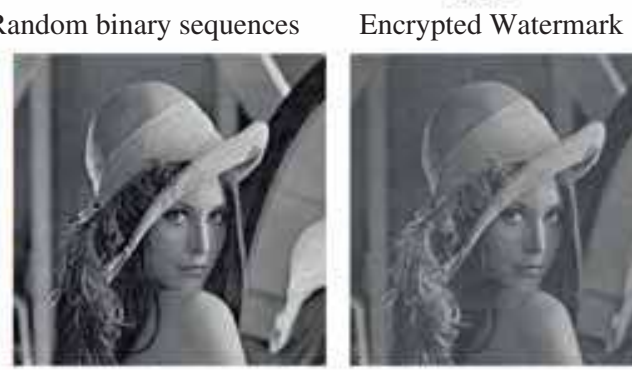

Watermarked Green Plane

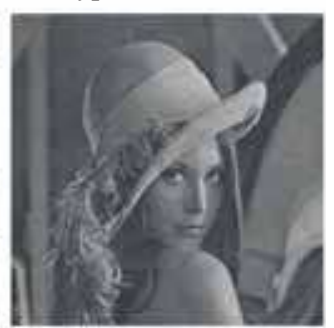

Watermarked Blue Plane
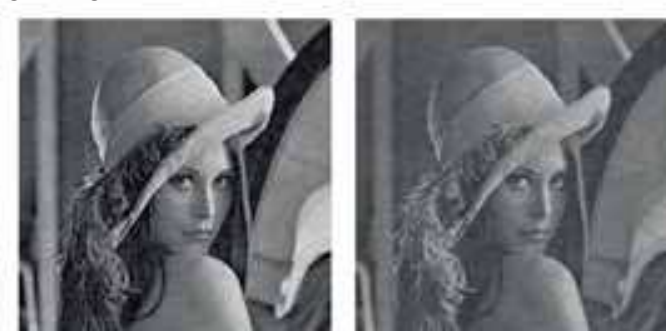

Watermarked Green Plane Watermarked Blue Plane

(c) Embedding strength $\alpha=8$

Figure 7. Imperceptibility test taking three different values of $\alpha$. 


\subsection{Imperceptibility test}

In this test we check whether the embedding is perceptually visible or not. Figure 7 shows the test results taking different values of $\alpha$ and demonstrates both self-encryption and random key encryption. It is clear by figure 7(a) that there is no perceptual trace of marked information on color watermarked image. The green component of watermarked image seems to be free from degradation and slight degradation is visible in blue plane due to watermark embedding. Figure 7(b) shows both the planes green and blue of watermarked image are free from degradation when $\alpha$ is taken as 3 . While in figure 7(c) it can be seen that the degradation of quality is perceptually visible in both green and blue planes of watermarked image if $\alpha$ is taken as 8 .

Original test images (a), (b) and (c) with embedded watermark

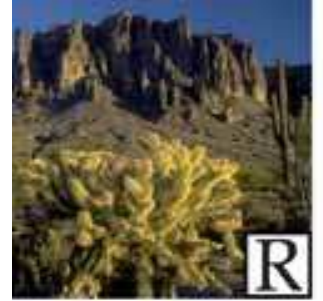

(a)

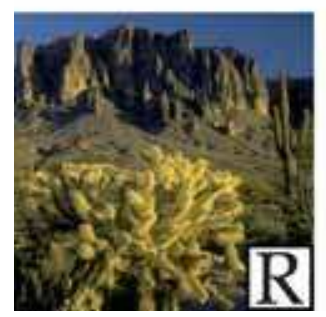

(d)

\subsection{PSNR and CC without attacks}

Figure 8 depicts original watermark, test images and respective watermarked images together with extracted watermark. It is quite clear that PSNR value for every watermarked image is equal. The reason behind this is two-way embedding mechanism that controls the PSNR value to remain constant. Two-way embedding ensures participation of every pixel in watermark embedding process. Due to this reason, presented method is capable to claim exact amount of additive noise due to watermark embedding. Additive noise in watermarked image can be calculated a priori with the help of image size in pixel and embedding strength $\alpha$. The $\mathrm{CC}$ value 1 for extracted watermark in the figure represents $100 \%$ similarity with original watermark.

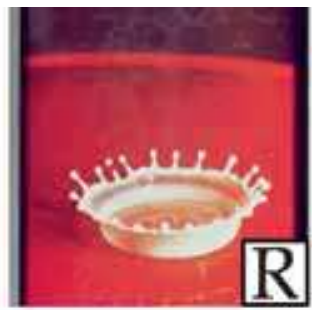

(b)

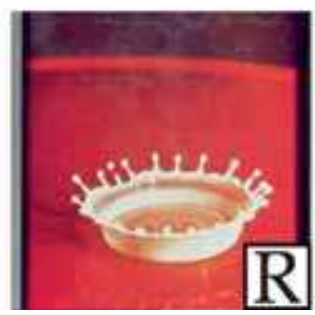

(e)

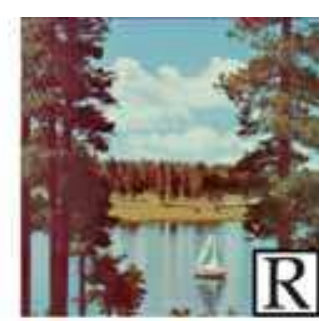

(c)

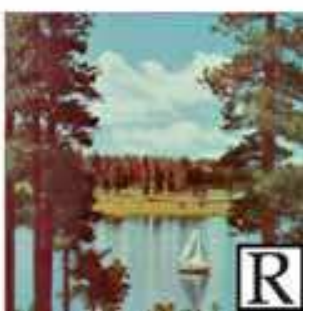

(f)
Figure 8. PSNR and $\mathrm{CC}$ values without attack.

Watermarked Images with embedded watermark

Applying $3 \times 3$ median filtering on (a), $5 \times 5$ median filtering on (b) and $7 \times 7$ median filtering on (c)

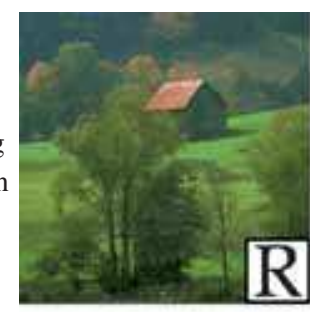

(a)

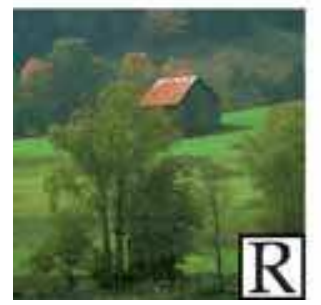

(d)

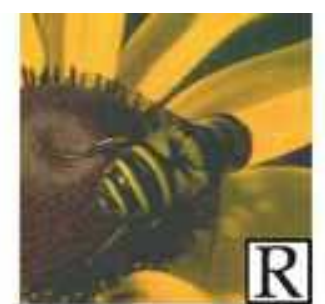

(b)

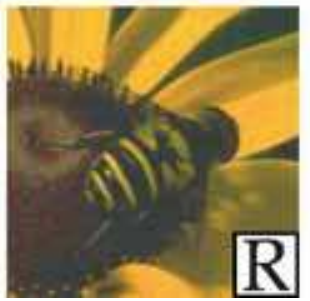

(e)

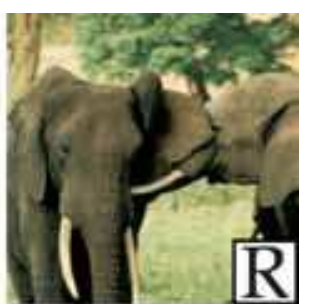

(c)

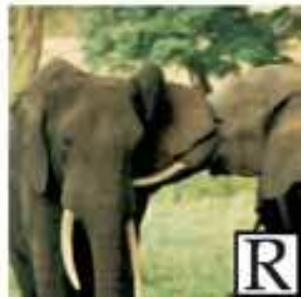

(f)
Figure 9. Median filtering attack. 


\subsection{Median filtering attack}

Median filtering attacks with mask size of $3 \times 3,5 \times 5$ and $7 \times 7$ are performed on watermarked version of test images. Figure 9 demonstrates the effects of median filtering on test images. In the figure it can be clearly seen that watermark is extracted without any error in $3 \times 3$ and $5 \times 5$ median filtering attacks, whereas in case of $7 \times 7$ median filtering slight degradation in extracted watermark is visible.

\subsection{Gaussian low pass filtering attack}

Gaussian low pass filtering attacks by taking mask sizes of $3 \times 3,5 \times 5$ and $7 \times 7$ are performed on watermarked test images and the results are depicted in figure 10. High value of CC shows that proposed method gives good performance against these attacks.

Watermarked Images with embedded watermark

Applying 3×3 Gaussian filtering on (a), $5 \times 5$ Gaussian filtering on (b) and $7 \times 7$

Gaussian filtering on (c)

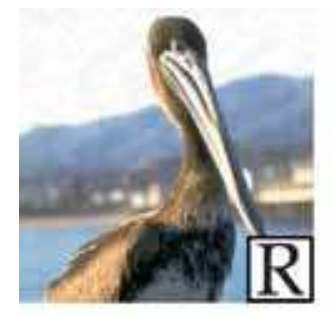

(a)

Attacked images and extracted watermark

(d) $\mathrm{PSNR}=25.9047$, $\mathrm{CC}=0.9961$

(e) $\mathrm{PSNR}=30.2040$, $\mathrm{CC}=0.9783$

(f) $\mathrm{PSNR}=26.4433$, $\mathrm{CC}=0.8957$

Watermarked Images with embedded watermark

Removing 20 random rows \& columns from (a), 50 random rows \& columns from (b) and 100 random rows \& columns from (c)

Attacked images and extracted watermark

(d) $\mathrm{PSNR}=19.9730$, $\mathrm{CC}=1$

(e) $\mathrm{PSNR}=16.5908$, $\mathrm{CC}=1$

(f) $\mathrm{PSNR}=12.2097$, $\mathrm{CC}=0.9192$

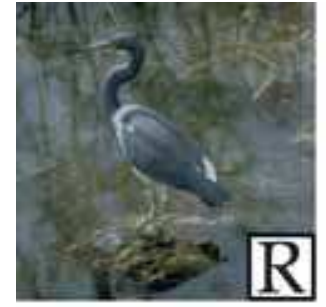

(a)

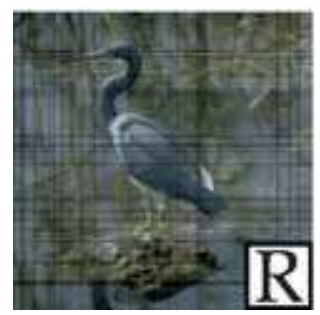

(d)

\subsection{Remove random line attack}

Number of random rows and columns are removed from the image in this attack. Method is tested against removal of 10 random rows \& 10 random columns, 20 random rows \& columns, 50 random rows \& columns and 100 random rows $\&$ columns. Figure 11 shows the results of method after removing 20, 50 and 100 random rows \& columns one by one. It is seen that proposed method resists this attack efficiently.

\subsection{Scaling attack}

Image resizing has been done for various scaling factors such as $25,50,75,125,150$ and 200 in percentage under this experiment. The value of $\mathrm{CC}$ is 1 for image scaling

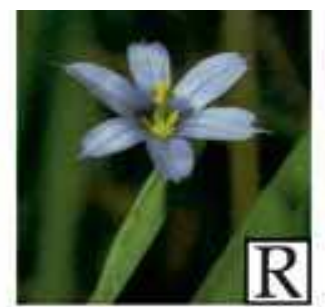

(b)

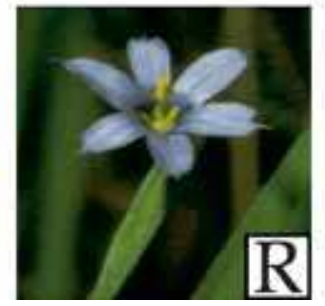

(e)

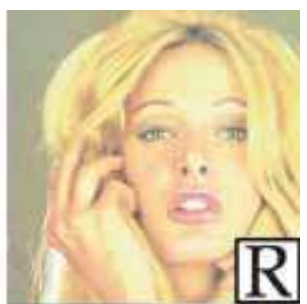

(c)

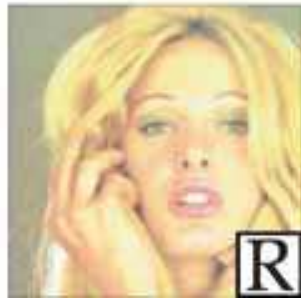

(f)

Figure 10. Gaussian low-pass filtering attack.

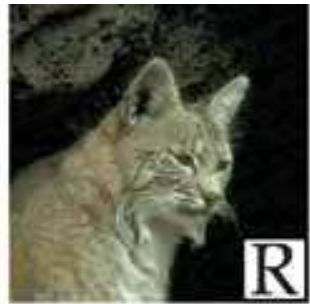

(b)

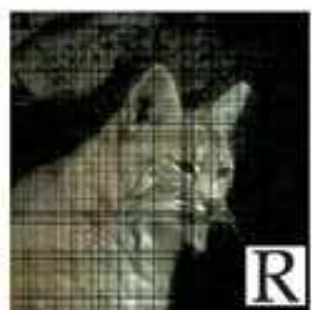

(e)

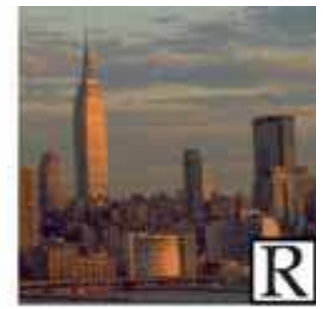

(c)

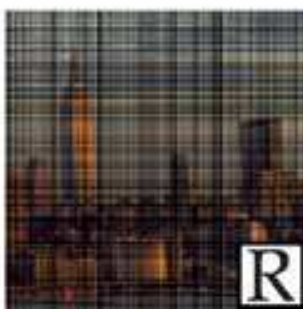

(f)

Figure 11. Removal of random rows \& columns. 
factors more than $100 \%$ and it is inside the range $0.9-1$ for factors less than $100 \%$ that shows proposed method works well against scaling attack. Figure 12 depicts the effects of scaling with factors 25, 50 and 200 on watermarked images. Before applying the extraction procedure images are rescaled to their original size.

\subsection{JPEG compression attack}

Loss compression attacks are most common and they can change the block intensity profile of image significantly [24]. JPEG compression removes low intensity information to achieve compression. Due to this reason, quality of image subsequently degrades and so the marked information degrades badly. The CC value for image watermarked with method proposed by Nasir et al [19] is less than 0.5 in JPEG
20 compression. Proposed method improves the results for CC values; as in case of JPEG 20, CC value touches 0.71 . This is due to the fact that method uses two component images green and blue for embedding, so it has two alternatives to extract marking information on bit level, in which it chooses more probable one. Figure 13 depicts the effects of JPEG compression on watermarked image.

\subsection{Cropping attack}

Multiple watermarking has shown good robustness against cropping attack as reported in Nasir et al [19]. That is why multiple watermarking is used in proposed scheme to achieve good performance against cropping. Even from $75 \%$ cropped image watermark can be extracted with $\mathrm{CC}$ value 1 . This is due to the fact that marked information is
Watermarked Images with embedded watermark

Resizing the image (a) with scaling factor $25 \%$, (b) with scaling factor $50 \%$, and (c) with scaling factor $200 \%$

Attacked images and extracted watermark

(d) PSNR $=26.1334$, $\mathrm{CC}=0.9235$

(e) $\mathrm{PSNR}=26.0781$, $\mathrm{CC}=0.9783$

(f) $\mathrm{PSNR}=34.1842$, $\mathrm{CC}=1$

Watermarked Images with embeddd watermark

Applying JPEG compression with quality factor of $80 \%$ on (a), with quality factor of $50 \%$ on (b) and with quality factor of $20 \%$ on (c)

Attacked images and extracted watermark

(d) PSNR $=30.6718$, $\mathrm{CC}=1$

(e) $\mathrm{PSNR}=23.6593$, $\mathrm{CC}=0.8806$

(f) $\mathrm{PSNR}=29.9026$, $\mathrm{CC}=0.7051$

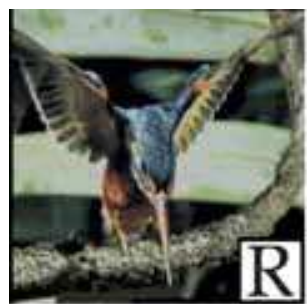

(a)

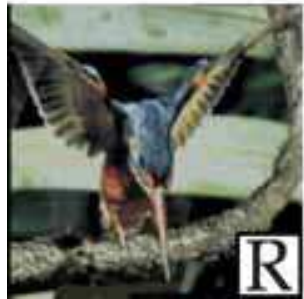

(d)

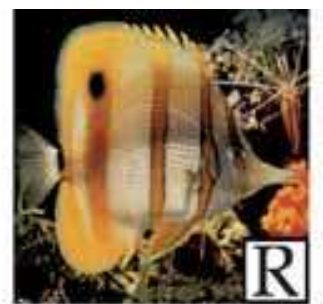

(b)

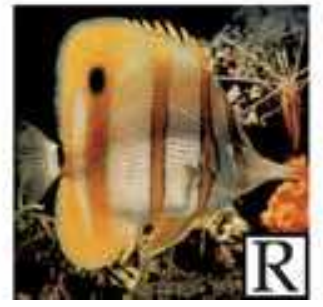

(e)

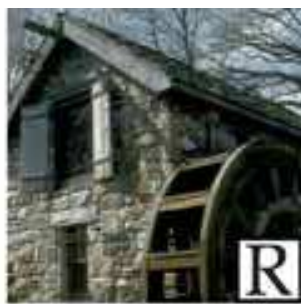

(c)

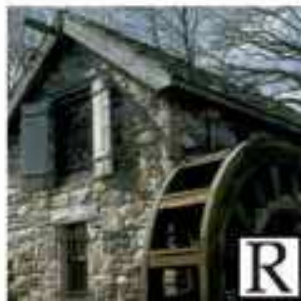

(f)
Figure 12. Scaling attack.

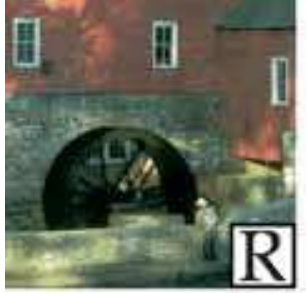

(a)

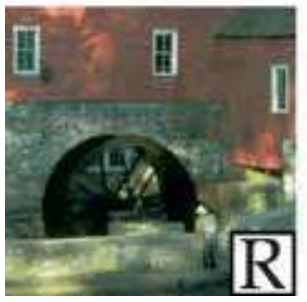

(d)

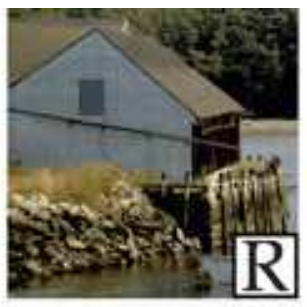

(b)

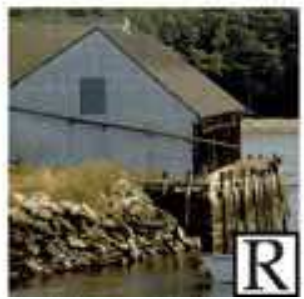

(e)

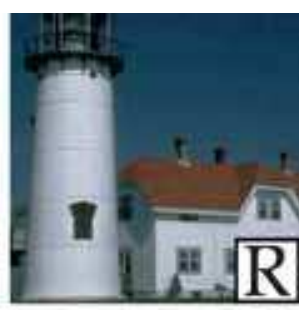

(c)

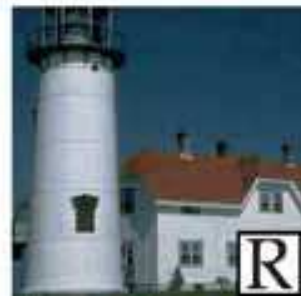

(f)
Figure 13. Loss compression attack. 
embedded in multiple regions of image and a complete watermark can be found if at least one fourth of image is available with exact positioning of marked regions. It is found that method also achieves very good results in random cropping of watermarked image if approximately half of image area is available. Figure 14 depicts the impact of cropping on watermarked images.

\subsection{Rotation, rotation scale and rotation crop attacks}

The limitation of non-blind spatial watermarking is that watermarked image should be in the same orientation and direction with original image e.g. it is necessary that a rotated watermarked image should be anti-rotated with same angle before extraction. In case of rotation attack, proposed method requires the angle of rotation to be known a priori. Most of the spatial watermarking methods can sustain basic manipulation but when combination of attacks is applied, they fail to give satisfactory results, as in case of rotation scale and rotation crop attacks. In rotation scale, watermark image first rotated with a slight angle then the resultant image which is slightly larger in size is rescaled to its original size. In rotation crop attack watermarked image is first rotated with a slight angle and then resultant image is cropped to its original size. Proposed method can handle these attacks efficiently, if rotation angle is known previously. Before applying extraction attacked image antirotated and rescaled to its original size. Experiments are carried out on rotation, rotation scale and rotation cropping taking the step size (angle) of $-2,-1,-0.5,0.5,1,2,30$, 45 and 90 degree. Anti-rotation is carried out by bilinear interpolation. Figure 15 depicts the effects of rotation, rotation-scale and rotation-crop attack.

\begin{abstract}
Watermarked Images with embedded watermark

Cropping of $50 \%$ image area from (a), $75 \%$ cropping from (b) and $45 \%$ random cropping from (c)
\end{abstract}

Attacked images and extracted watermark

(d) $\mathrm{PSNR}=9.9806$, $\mathrm{CC}=1$

(e) PSNR = 7.8917, $\mathrm{CC}=1$

(f) $\mathrm{PSNR}=10.5864$, $\mathrm{CC}=0.8651$

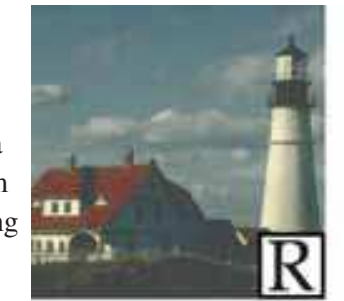

(a)

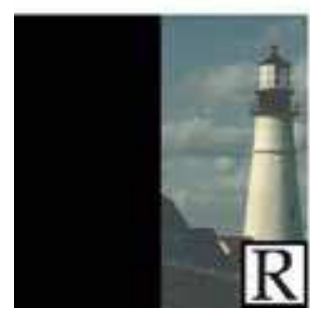

(d)

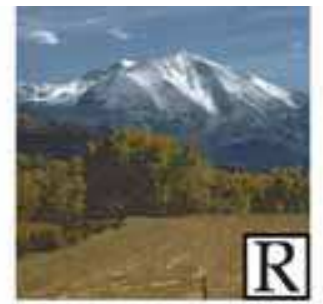

(b)

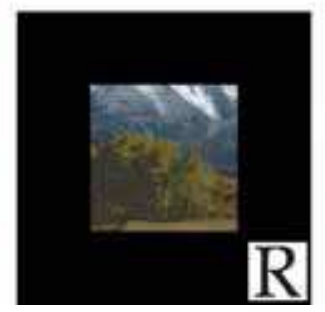

(e)

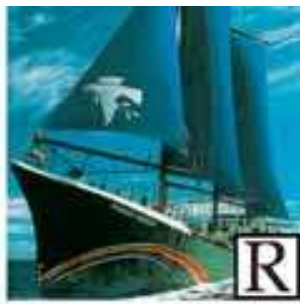

(c)

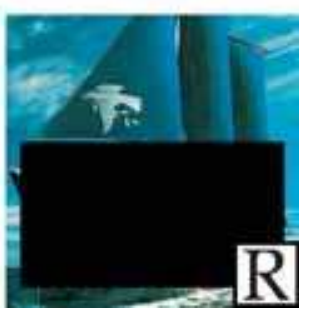

(f)

Figure 14. Cropping attack.

Watermarked Images with embedded watermark

Applying rotation on image (a) with angle 30 degree, rotate scale on image (b) with 5 degree and Rotate- crop on image (c) with 45degree

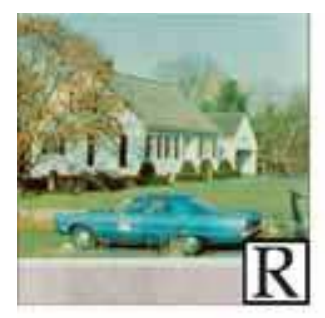

(a)

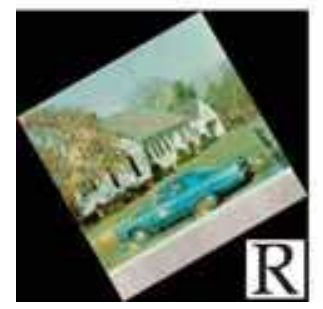

(d)

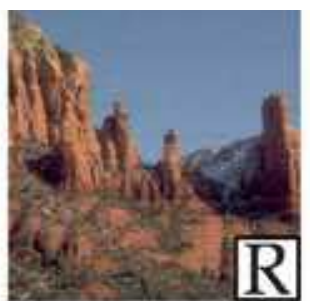

(b)

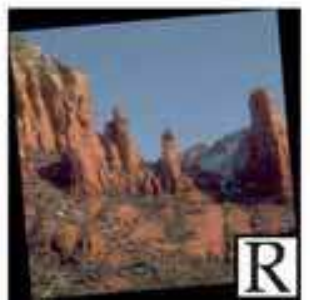

(e)

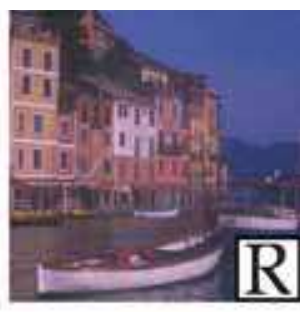

(c)

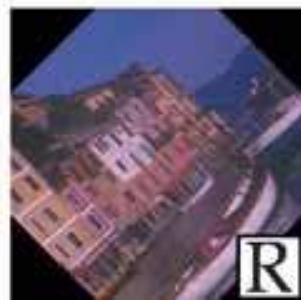

(f)

Figure 15. Effects of rotation, rotation-scale and rotation-crop. 
Watermarked Images with embedded watermark

Adding 50\% random salt and pepper noise in image (a), $60 \%$ noise in image (b) and $80 \%$ noise in image (c)

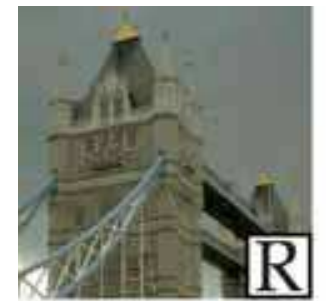

(a)

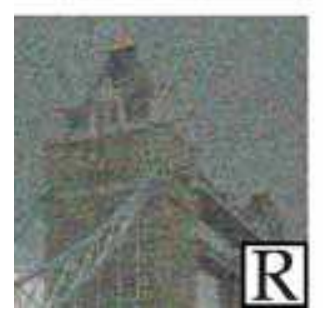

(d)

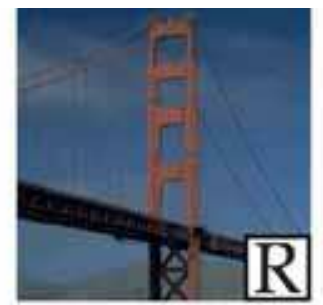

(b)

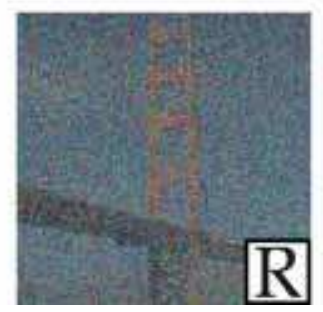

(e)

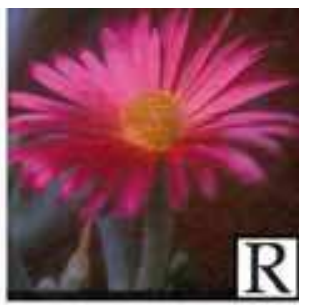

(c)

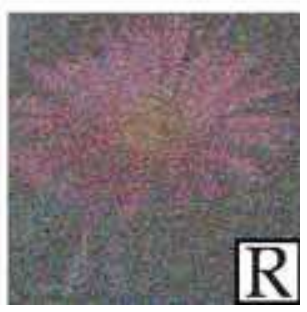

(f)

Figure 16. Addition of random noise.

Table 2. Comparison of Proposed method with the method proposed by Nasir.

\begin{tabular}{|c|c|c|c|c|c|c|}
\hline \multirow[b]{2}{*}{ Attacks } & \multicolumn{2}{|c|}{ Lena $(\mathrm{CC})$} & \multicolumn{2}{|c|}{ Peppers (CC) } & \multicolumn{2}{|c|}{ Baboon $(\mathbf{C C})$} \\
\hline & Proposed method & Nasir's method & Proposed method & Nasir's method & Proposed method & Nasir's method \\
\hline Free of attack & 1.0 & 1.0 & 1.0 & 1.0 & 1.0 & 1.0 \\
\hline Remove lines 10 & 1.0 & 0.99 & 1.0 & 0.99 & 1.0 & 0.98 \\
\hline Remove lines 50 & 1.0 & 1.0 & 1.0 & 0.97 & 1.0 & 0.95 \\
\hline Remove lines100 & 0.8601 & 0.99 & 0.8839 & 0.96 & 0.8719 & 0.94 \\
\hline JPEG 80 & 1.0 & 0.99 & 0.9980 & 0.98 & 0.9961 & 0.96 \\
\hline JPEG 50 & 0.9842 & 0.91 & 0.9469 & 0.88 & 0.9054 & 0.85 \\
\hline JPEG 20 & 0.6740 & 0.47 & 0.6287 & 0.48 & 0.5909 & 0.49 \\
\hline Scaling 50\% & 1.0 & 1.0 & 1.0 & 0.98 & 0.9312 & 0.92 \\
\hline Scaling $200 \%$ & 1.0 & 1.0 & 1.0 & 0.99 & 1.0 & 0.99 \\
\hline Rotation-scale 0.5 & 0.9744 & 0.87 & 0.9921 & 0.82 & 0.9232 & 0.73 \\
\hline Rotation-scale 1 & 0.9803 & 0.60 & 0.9902 & 0.66 & 0.9214 & 0.46 \\
\hline Median filter $3 \times 3$ & 1.0 & 0.99 & 1.0 & 0.99 & 1.0 & 0.83 \\
\hline Median filter $5 \times 5$ & 0.9961 & 0.99 & 0.9980 & 0.98 & 0.9134 & 0.89 \\
\hline Median filter $7 \times 7$ & 0.9547 & 0.99 & 0.9416 & 0.95 & 0.7333 & 0.77 \\
\hline Crop $25 \%$ & 1.0 & 1.0 & 1.0 & 1.0 & 1.0 & 1.0 \\
\hline Rotation $2^{\circ}$ & 0.9803 & 1.0 & 0.9941 & 0.91 & 0.9332 & 0.85 \\
\hline Rotation $5^{\circ}$ & 0.9744 & 0.99 & 0.9882 & 0.91 & 0.9846 & 0.79 \\
\hline Rotation $45^{\circ}$ & 0.9803 & 0.99 & 0.9863 & 0.94 & 0.9468 & 0.83 \\
\hline Rotation $90^{\circ}$ & 1.0 & 1.0 & 1.0 & 0.96 & 1.0 & 0.84 \\
\hline Gaussian filter $3 \times 3$ & 0.9961 & 1.0 & 0.9961 & 0.65 & 0.8976 & 0.97 \\
\hline
\end{tabular}

\subsection{Addition of random noise attack}

In this category of attack random noise is introduced to watermarked images. The results are shown for the random salt and pepper noise with density level of $0.5,0.6$ and 0.8 in figure 16. Experiments are carried out from taking density 0.1 to 0.8 of noise and in every case the $\mathrm{CC}$ value of extracted watermark is near to 1 that shows proposed method can withstand random noise addition very efficiently.

Finally comparison has been made between proposed method and the method proposed by Nasir et al [19] in table 2. Table 2 contains $\mathrm{CC}$ results found in various test attacks for proposed watermarking together with the $\mathrm{CC}$ results of method proposed by Nasir et al [19]. For comparison, standard test images Lena, Peppers and Baboon of the size 512 $\times 512$ are taken from SIPI image database [25]. Comparison results suggest that the proposed method performs better against a number of processing attacks.

\section{Conclusion}

The performance of watermarking method in terms of added noise varies for different images. The cause of this problem is modifying the pixel intensities having values on or near range (0 or 255$)$ to go beyond. Two-way mechanism 
proposed in this paper is the best possible solution for this problem in which pixel intensity is modified after a careful check whether the value should be incremented or decremented. That is why proposed method can claim a certain amount of additive noise to every possible image, if size of the image and embedding strength is taken as constant. Multiple watermarking in two components of host image makes proposed method more robust and more imperceptible. Self-encryption in proposed method makes owner of image; free from the burden of supplying and remembering the secret keys. Stirmark benchmark 4.0 is used to test proposed method against various attacks and experimental results shows that method is highly robust.

\section{References}

[1] Song C, Sudirman S, Merabti M and Jones D L 2010 Analysis of digital image watermark attacks. 7th IEEE Conference on Consumer Communications and Networking (CCNC), pp. 1-5

[2] Yeung M M 1998 Digital watermarking. Communication of the ACM 41(7): 31-33

[3] Nikolaidis N and Pitas I 1996 Copyright protection of images using robust digital signatures. In: ICASSP-96. IEEE International Conference on Acoustics, Speech, and Signal Processing, vol. 4, pp. 2168-2171

[4] Kim B S, Choi J G, Park C H, Won J U, Kwak D M, Oh S K, Koh C R and Park K H 2003 Robust digital image watermarking method against geometrical attacks. Real-Time Imaging 9(2): 139-149

[5] Darmstaedter V, Delaigle J F, Quisquater J J and Macq B B 1998 Low cost spatial watermarking. Comput. Graph. 22(4): 417-424

[6] Zaho X and Ho A T S 2010 An introduction to robust transform based image watermarking technique from intelligent multimedia analysis for security applications. Studies in computational intelligence 282: 337-364

[7] Sánchez D, Orfila A, Hernández J and Sierra J 2001 Robust new method in frequency domain watermarking, from information security, Lecture Notes in Computer Science, Springer, vol. 2200, pp. 166-182

[8] Raval M S and Rege P P 2003 Discrete wavelet transform based multiple watermarking scheme. In: TENCON 2003 Conference on Convergent Technologies for Asia-Pacific Region, vol. 3, pp. 935-938

[9] Cheng L M, Cheng L L, Chan C K and Ng K W 2004 Digital watermarking based on frequency random position insertion. Control, Automation, Robotics and Vision Conference 8th, ICARCV, 2004, vol. 2, pp. 977-982
[10] Bhatnagar G and Raman B 2009 A new robust reference logo watermarking scheme. Multimedia Tools Appl 52(2): 621-640

[11] Chavan S, Shah R, Poojary R, Jose J and George G 2010 A novel robust colour watermarking scheme for colour watermark images in frequency domain. International Conference on Advances in Recent Technologies in Communication and Computing (ARTCom), pp. 96-100

[12] Chan C K and Cheng L M 2004 Hiding data in images by simple LSB substitution. Pattern Recognit 37(3): 469-474

[13] Nikolaidis N and Pitas I 1998 Robust image watermarking in the spatial domain. Signal Process 66(3): 385-403

[14] Maity S P and Kundu M K 2002 Robust and blind spatial watermarking in digital image. In: Proceedings of $3 \mathrm{rd}$ Indian Conference on Computer Vision, Graphics and Image Processing (ICVGIP), pp. 388-393

[15] Kimpan S, Lasakul A and Chitwong S 2004 Variable block size based adaptive watermarking in spatial domain. In: ISCIT 2004 IEEE International Symposium on Communications and Information Technology, vol. 1, pp. 374-377

[16] Lopes I O, Barcelos C A Z, Batista M A and Silva A M 2006 Enhanced watermarking scheme based on texture analysis, Lecture Notes in Computer Science Springer, vol. 4179, pp. 746-756

[17] Verma B, Jain S, Agrawal D and Phadikar A 2006 A new color image watermarking scheme. INFOCOMP J. Comput. Sci. 5(2): 37-42

[18] Cika P 2007 New watermarking scheme for colour image. In: Proceedings of International Federation for Information Processing IFIP, vol. 245, pp. 497-504

[19] Nasir I, Weng Y and Jiang J 2010 Multiple spatial watermarking technique in color images. Signal, Image Video Process 4(2): 145-154

[20] Liu V, R h Li and Gao Q 2004 A new watermarking method based on DWT green component of color image. In: Proceedings of 2004 International Conference on Machine Learning and Cybernetics, vol. 6, pp. 3949-3954

[21] CVG-UGR test image database at http://decsai.ugr.es/cvg/ dbimagenes/c512.php

[22] Petitcolas F A P, Anderson R J and Kuhn M G 1998 Attacks on copyright marking systems. In: Proceedings of Information Hiding, 2nd International Workshop, Lecture Notes in Computer Science Springer, vol. 525, pp. 218-238

[23] Okada M, Okabe Y and Uehara I 2009 Semi-blind fingerprinting utilizing ordinary existing watermarking techniques. In: Proceedings of 8th international workshop on digital watermarking (IWDW), Guildford, U K

[24] Mukherjee D P, Maitra S and Acton S T 2004 Spatial domain digital watermarking of multimedia objects for buyer authentication. IEEE Trans. Multimedia. 6(1): 1-15

[25] SIPI image database- http://sipi.usc.edu/database/database. php?volume $=$ misc 